\title{
UNA ENCUESTA PARALIZADA EN EL TIEMPO: EL CUADERNO DE PREPARACIÓN DE LECCIONES
}

\section{A survey paralyzed in time: the lesson preparation notebook}

\author{
Rosa Ortiz De Santos \\ Universidad de Valladolid \\ Correo-e: rosa.ortiz@uva.es
}

Recepción: 23 de julio de 2020. Envío a informantes: 31 de agosto de 2020 Aceptación definitiva: 28 de noviembre de 2020

Resumen: En 1932 la revista Escuelas de España publicó las respuestas de una veintena de maestros a una encuesta sobre el cuaderno de preparación de lecciones. Informaba de que, más adelante, compartirían el análisis y las conclusiones del estudio. La desaparición temporal de la revista provocó que estos datos no llegasen a ser objeto de análisis. En esta propuesta, siendo conocedores de la evolución que vivieron los cuadernos de preparación, damos continuidad a la investigación. Los resultados evidencian el valor que los docentes encuestados otorgaban a los cuadernos como un recurso que favorecía la autonomía y el empoderamiento del profesorado. Al mismo tiempo, de manera casi premonitoria, temían que llegasen a utilizarse como un instrumento de control de la actividad docente.

Palabras clave: cuaderno de preparación de lecciones; Escuelas de España; prensa pedagógica; planificación educativa; programa de enseñanza.

AвsтRAct: In 1932, the journal Escuelas de España published the answers of twenty teachers to a survey about the "cuaderno de preparación de lecciones». It reported that they would later share the analysis and conclusions of the study. The temporary disappearance of the journal meant that this data was not analysed. In this proposal, being aware of the evolution that the preparation notebooks underwent, we give continuity to the research. The results show the value that the teachers surveyed gave to the notebooks as a resource that favoured the autonomy and empowerment of teachers. At the same time, almost predictably, they feared that they would be used as an instrument to control the teacher's activity. 
KEY WORDs: lesson preparation notebook; Escuelas de España; pedagogical press; educational planning; teaching programme.

\section{Introducción}

$\mathrm{E}$

L PRIMER TERCIO DEL SIGLO XX fue una época de gran riqueza en lo pedagógico y lo cultural; tanto es así, que desde la historiografía se ha denominado a esta etapa la edad de plata de la cultura española. En esta época, en la que se pretendía lograr la regeneración de España, se consideró prioritaria la renovación de la educación, dado que existía el convencimiento de que era el motor de cambio de las restantes esferas de la sociedad ${ }^{1}$. Ahora bien, la transformación educativa tuvo su auge con la llegada de la Segunda República. Durante el quinquenio republicano, decía Pérez Galán², nuestro país vivió un periodo de florecimiento pedagógico sin precedentes, que se desarrolló bajo una clara influencia de los principios de la Institución Libre de Enseñanza (ILE) y de los ideales defendidos por el Partido Socialista Obrero Español (PSOE) que, a su vez, se había nutrido de la anterior. Del mismo modo, el pensamiento pedagógico de la corriente internacional de la Escuela Nueva penetró en el ideario de una buena parte del magisterio ${ }^{3}$. La brillantez pedagógica de la educación republicana ha generado un gran interés a los investigadores; ya contamos con numerosos manuales básicos de referencia ${ }^{4}$.

Los estudios generales de la época se han venido intercalando con otras investigaciones de temáticas específicas. Así, a vuelapluma, podemos citar varios temas: biografías de maestros y maestras y de otras personalidades señeras de la pedagogía española; iniciativas de renovación pedagógica vinculadas a diferentes instituciones (Misiones Pedagógicas, colonias escolares de vacaciones, pensiones de estudio de la JAE, etc.); centros educativos de referencia (Cervantes, Príncipe de Asturias, Baixeras, etc.); títulos de prensa pedagógica profesional (Revista de Pedagogía, Escuelas de España, Revista de Escuelas Normales, etc.); experiencias de renovación pedagógica locales, regionales y nacionales; principios y métodos pedagógicos; o las diferentes disciplinas escolares, entre muchos otros.

De Puelles Benítez, Manuel: Modernidad, republicanismo y democracia: una historia de la educación en España (1898-2008), Valencia, Tirant lo Blanch, 2009.

2 Pérez Galán, Mariano: La enseñanza en la Segunda República española, Madrid, Edicusa, 1975; Pérez Galán, Mariano: "La enseñanza en la Segunda República», Revista de Educación, n. ${ }^{\circ}$ extraordinario (2000), pp. 317-332.

3 Del Pozo Andrés, María del Mar: «La escuela nueva en España: crónica y semblanza de un mito", Historia de la Educación, n. ${ }^{\circ} 22-23$ (2004), pp. 317-346.

4 Pérez Galán, Mariano: La enseñanza en..., op. cit.; Molero Pintado, Antonio: La reforma educativa de la Segunda República Española. Primer bienio, Madrid, Santillana, I977; SAMAnieGo Boneu, Mercedes: La política educativa de la II República durante el bienio azañista, Madrid, CSIC, 1977; Lozano SeIjas, Claudio: La educación republicana, Barcelona, Universidad de Barcelona, I980; PÉrez GaláN, Mariano: «La enseñanza en...», op. cit. 
Otro tema de interés emergente en la investigación en historia de la educación ha sido el estudio de la cultura material de la escuela ${ }^{5}$. Este ámbito de la cultura escolar implica el análisis de los objetos materiales, como fuente y como objeto de estudio, atendiendo a aspectos como su «función, uso, distribución en el espacio, materialidad, simbología, su Introducción, [...] y desaparición o abandono.... ${ }^{6}$. Los diarios y los cuadernos del profesorado forman parte de esa cultura material, que nos permite «conocer el funcionamiento intrínseco de una institución o la labor de un docente» ${ }^{7}$. En esta aportación se pretende contribuir a la investigación de la cultura material de la escuela mediante el análisis de los cuadernos de preparación de lecciones, como objeto de estudio, a partir de la revisión de prensa periódica de diferentes momentos históricos, y a través de las opiniones que un nutrido grupo de maestros republicanos vertió en una revista pedagógica profesional.

\section{I. «Cada maestrillo tiene su librillo»: los cuadernos de preparación de lecciones}

El cuaderno de preparación de lecciones era un documento en el que cada docente, de forma individual, plasmaba sucintamente el diseño de su intervención educativa, prestando atención a todos los elementos del proceso educativo y de la actividad del aula. Se trataba pues, de «un esquema-guía orientado a facilitar su desarrollo, personalizando y adaptando los programas a la realidad específica de cada escuela y a los usos y costumbres del docente» ${ }^{8}$, es decir, consistía en un modo de adaptar los contenidos a la realidad y las características particulares de cada aula. Antonio Viñao propone una taxonomía de los relatos autobiográficos de los docentes. Dentro de los «diarios» sitúa los cuadernos de preparación de lecciones y los describe como una de las fuentes documentales «que más se acerca y nos aproxima $[\ldots]$ a las prácticas o realidad cotidiana del aula» ${ }^{9}$, y dice de ellos que eran como un "planning', agenda prospectiva o memoria del futuro» ${ }^{10}$, que se realizaba de forma previa a las sesiones de aula.

SANCHIDRIÁn BLANCo, Carmen: «La historia de la cultura material de la educación: un campo de investigación emergente», en Vila Merino, E. S. y Grana Gil, I. (coords.): Investigación educativa y cambio social, Barcelona, Octaedro, 2020.

6 Sanchidrián Blanco, Carmen: La historia cultural de la educación: entre cambios y continuidades, Málaga, SPICUM, 20I3, p. I5.

7 Ibidem, p. I7.

8 López Martín, Ramón: «La figura del maestro y la cultura pedagógica en la España franquista. Reflejos desde la literatura», Utopía y Praxis Latinoamericana, vol. 24, n. ${ }^{\circ} 87$ (2019), pp. 97II2, p. IO2.

9 Viñao Frago, Antonio: «Relatos y relaciones autobiográficas de profesoras y maestros», en Escolano Benito, A. y Hernández Díaz, J. M. (coords.): La memoria y el deseo. Cultura de la escuela y educación deseada, Valencia, Tirant lo Blanch, 2002, pp. 135-175, p. 150.

ro Ibidem, p. I50. 
El citado autor afirma que, aunque no era obligación de los docentes desarrollar de forma sistemática y diaria un cuaderno de preparación de lecciones, en gran parte de los manuales de pedagogía de comienzos del siglo xx se recomendaba su utilización y se ofrecían modelos y esquemas preestablecidos que podrían orientar a los educadores. Tal era su consideración que encontramos algunos artículos al respecto en títulos de prensa pedagógica profesional de la época. En 1932, el maestro de Huesca Fernando San Martín explica en El Magisterio Español que el cuaderno de preparación de lecciones era «como un índice del trabajo realizado en la Escuela en un día, en una semana, en un mes y en un curso. Será un diario de clases si se quiere. [...] Viene a ser el programa» ${ }^{11}$.

En 1933, en el mismo periódico, encontramos un extenso artículo, escrito por el maestro de Tinieblas de la Sierra (Burgos), José Andrés y Martín, acerca de estos cuadernos. En dicho artículo se defendía el empleo del instrumento como un medio eficaz para luchar contra la improvisación. El autor opinaba que los cuadernos de preparación habían de fundamentarse en tres principios pedagógicos. El primero era el «rigor lógico», que garantizase una labor seria, asentada sobre un plan racional adecuado a las características del contexto. El segundo consistía en «disponer el espíritu del niño», es decir, elaborar un cuaderno basado en métodos activos y en un procedimiento intuitivo; el autor citaba a Pestalozzi para defender esta idea. El tercero y último hacía alusión al «equilibrio entre la teoría y la práctica», entre el «saber» y el «hacer». Tras la exposición de estos principios, explicaba cómo se debía elaborar un cuaderno de preparación de lecciones, partiendo de que «su uso no puede [...] acomodarse a reglas fijas e inflexibles, porque sería matar la iniciativa y personalidad del maestro» ${ }^{12}$. Para el autor, el cuaderno de preparación de lecciones era un valioso auxiliar que favorecía la «Escuela renovada, activa [y] moderna en su espíritu» ${ }^{13}$.

Este ánimo progresista y de carácter renovador que se otorgó a los cuadernos en el primer tercio del siglo xx vivió una transformación. Casi finalizada la Guerra Civil española, por la Orden de 20 de enero de 1939, se comenzó a exigir a los maestros del nacionalcatolicismo llevar al día el cuaderno de preparación de lecciones. En el artículo 9 de la citada Orden se dictaba que «... todo Maestro llevará un cuaderno de preparación de lecciones, de conformidad con el programa de la Escuela y orientaciones que el Inspector le señale» ${ }^{14}$. En este contexto llegó a considerarse "como un elemento de control de la actividad del docente ${ }^{15} \mathrm{y}$ de control ideológico. De hecho, y continuamos citando la normativa, los inspectores de educación debían encargarse de recoger en la Memoria que mensualmente

"SAn Martín, Fernando: «De nuestro concurso. Un archivo escolar», El Magisterio Español, n. ${ }^{\circ}$ 9063, 17, noviembre, 1932, pp. 313-315. Recuperado de https://cutt.ly/AakHD3y, p. 314 .

${ }_{12}$ Andrés y Martín, José: «De nuestro concurso. Los diarios de clase», El Magisterio Español, n. ${ }^{\circ}$ 9IIO, 9, marzo, 1933, pp. 462-466. Recuperado de https://cutt.ly/XakAub7, p. 463.

${ }_{13} \quad$ Ibidem, p. 462.

${ }^{14}$ Boletín Oficial del Estado, n. ${ }^{\circ} 27,27$ de enero de 1939. Recuperado de https://cutt.ly/ ByDYh3z, p. 493.

is López Martín, Ramón: op. cit., p. 102. 

ROSA ORTIZ DE SANTOS

enviaban a la Jefatura del Servicio Nacional de Primera Enseñanza «datos relacionados con el cuaderno de clase de los niños y del cuaderno de preparación de clase del Maestro» ${ }^{16}$.

La obligatoriedad de estos diarios formales fue noticia en la prensa de la época. En marzo de 1939 la inspectora Paquita Montilla escribió en Azul. Órgano de la Falange Española de las JONS un artículo en el que justificaba la obligatoriedad del cuaderno de preparación de lecciones. Opinaba, tajantemente, que «el trabajo escolar no admite improvisaciones. Por culto que sea un maestro, por recursos didácticos que posea, si se lanza a su labor sin meditar primero sobre el propósito que debe animarla, y no ordena, ni prevé, ni delimita, aún inteligente y preparado, fracasará...» ${ }^{17}$.

Recalcaba que la elaboración de este cuaderno no suponía un recargo o un esfuerzo extra en las tareas del docente. Definió el cuaderno como «una síntesis clara y precisa del trabajo diario» ${ }^{18}$, que debía ajustarse a los tiempos escolares. Tan importante como el trabajo previo en el cuaderno, lo era la reflexión posterior, según la inspectora. Acaba su escrito afirmando que «... esa tendencia evidentemente realista y práctica y fecunda, al servicio de la Escuela, [...] [contribuye a hacer] de los niños católicos sinceros y patriotas excelentes» ${ }^{19}$. Esta última afirmación evidencia el control ideológico que pudo existir a través de los cuadernos de preparación.

En febrero de 1940 El Defensor Escolar publicó un artículo sobre los cuadernos de preparación de lecciones. Explicaba que, tiempo atrás, como ya hemos avanzado, el desarrollo de estos cuadernos era una opción voluntaria, si bien era considerado un mérito para los maestros y las maestras. «Su orientación, y la sinceridad destacada en sus páginas, eran la mejor demostración de la cultura pedagógica y de la organización escolar actuantes en la enseñanza primaria» ${ }^{20}$. Sin embargo, el autor afirmó que en la mayoría de las escuelas no se realizaba tal cuaderno. La realidad imperante era otra, pues se habían convertido, de forma legal, en obligatorios.

No basta con que el maestro diga: yo preparo las lecciones como siempre o yo sé la marcha de mi escuela y los niños 'van aprendiendo'. Es necesario que esa preparación se haga ordenada siguiendo un plan y desarrollando un programa estudiado con todo detalle en los que tendrán cabida cuantas lecciones ocasionales puedan complementar la formación cultural y la educación religiosa y patriótica de los alumnos ${ }^{21}$.

16 Boletín Oficial del Estado, op. cit., p. 493.

17 Montilla, Paquita: «Normas didácticas. El cuaderno de preparación de lecciones», Azul. Órgano de la Falange Española de las JONS, n. ${ }^{\circ}$ 760, 16, marzo, 1939, p. 9. Recuperado de https:// cutt.ly/8abvWvM, p. 9 .

I8 Ibidem, p. 9.

19 Ibidem, p. 9.

${ }_{20}$ El Defensor Escolar: «El cuaderno de preparación de lecciones y la libreta de síntesis», El Defensor Escolar, n. ${ }^{\circ}$ 196, 17 de febrero de 1940, pp. I-2. Recuperado de https://cutt.ly/Eabgo3x, p. I.

${ }^{21}$ Ibidem, p. I. 
En 1941, en La Escuela Española, se publicó otro artículo sobre el cuaderno de preparación de lecciones. El autor reflexionaba sobre la conveniencia de preparar las lecciones. Analizaba los obstáculos que algunos docentes encontraban en esta labor, ya que «no todos veían positiva esta tarea complementaria a la jornada laboral» ${ }^{22}$ :

Los reacios suelen escudarse en la falta de tiempo o en la sobra de preocupaciones; [...] Están, además, la desgana de los que trabajan sin vocación, el desconocimiento de los que enseñan sin técnica y la falta de hábito que, en esta empresa, como en todas, suele hacer difíciles las cosas, aunque en realidad no lo sean ${ }^{23}$.

En su opinión, «cuando uno trabaja con gusto, no mide el sacrificio, porque no le ve; solo tiene en cuenta el beneficio que tal trabajo produce. Y esta última consideración es para el maestro una fuente inagotable de placer» ${ }^{24}$. Acompañando a su discurso, encontramos una ficha-modelo orientativa para los maestros noveles o inexpertos:

FiguRA I. Ficha-modelo para la preparación de lecciones prácticas.

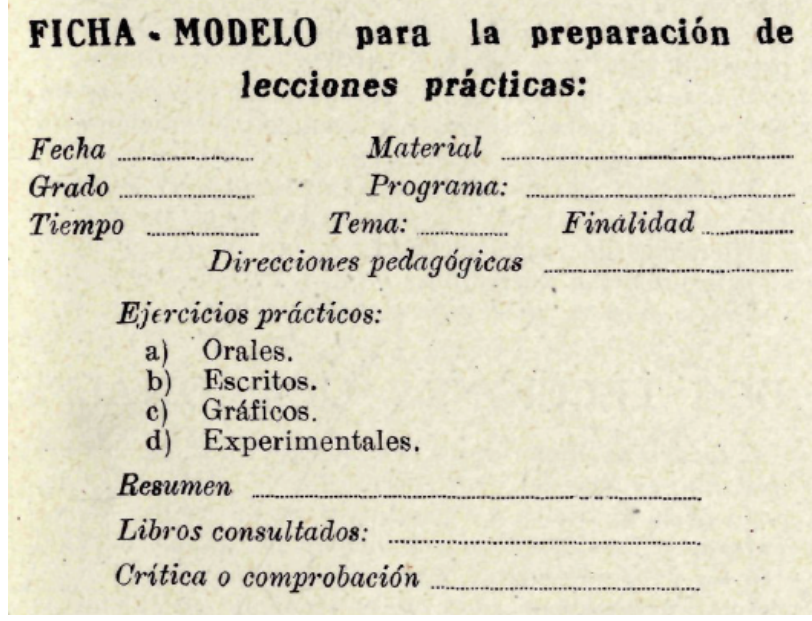

Fuente: Escuela Española, 25 de septiembre de $1941^{25}$.

22 Sanchidrián Blanco, María del Carmén y Gallego García, María del Mar: «Los cuadernos escolares como fuente y tema de investigación en Historia de la Educación», en BERRUEzo Albéniz, M. R. y Conejero López, S. (coords.): El largo camino hacia una educación inclusiva: la educación especial y social del siglo XIX a nuestros dias: XV Coloquio de Historia de la Educación, Navarra, Universidad Pública de Navarra, 2009, pp. 760-780, p. 772.

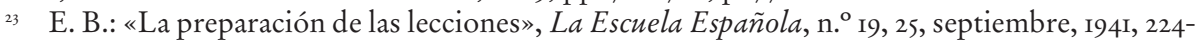
225. Recuperado de https://cutt.ly/labTqRP, p. 224.

24 Ibidem, p. 225.

${ }_{25}$ Ibidem, p. 225. 
En suma, en estos tiempos

... cada maestro dejaba constancia en el cuaderno de preparación de lecciones de su impronta personal, no sólo en la forma de diseñar y desarrollar el contenido de los temas y ejercicios sino en la forma de ilustrarlos, conscientes de que su saber y capacidad creativa habrían de ser rigurosamente valorados y sancionados, más tarde, por la Inspección de Enseñanza Primaria. Ese componente de originalidad, de intuición creativa, que daba un carácter personal e irrepetible a cada cuaderno del profesor, es lo que explica el dicho tan común en la época de «cada maestrillo tiene su librillo» ${ }^{26}$.

Este mismo autor afirma que fue con la Ley General de Educación (I970) cuando se pasó del cuaderno de preparación de lecciones a un modelo de programación didáctica más parecido al que se utiliza en la actualidad en el contexto educativo.

\section{La encuesta de Escuelas de España. ¿Cuál es tu opinión sobre el cuaderno de preparación de lecciones?}

\section{I. La prensa pedagógica como fuente de investigación: Escuelas de España}

«Entre las décadas de 1960 y I970, el uso de la prensa pedagógica como fuente para la escritura de la historia fue tomada con desconfianza y hasta desacreditada» ${ }^{27}$. Sin embargo, hoy en día los investigadores en historia de la educación la reconocen como un valioso aliado, llegando a definirla como «expresión viva y directa de todo lo que germina y aflora en el amplio campo de la educación, que es extraordinariamente rico y diverso, tanto como lo son las sociedades en el pasado, y sus procesos de formación» ${ }^{28}$.

La edad de plata de la cultura española, es decir, el periodo comprendido entre 1900 y 1936, fue una época álgida en la publicación de títulos de prensa pedagógica ${ }^{29}$. Escuelas de España fue una de las revistas publicadas en este periodo histórico. La obra pedagógica fue fruto de las inquietudes profesionales de tres audaces maestros que, en ese momento, desarrollaban su labor en la escuela rural segoviana: Pablo de Andrés Cobos, David Bayón Carretero y Norberto Hernanz

26 Parra Ortiz, José María: «La evolución de la enseñanza primaria y del trabajo escolar en nuestro pasado histórico reciente», Tendencias Pedagógicas, n. ${ }^{\circ}$ I4 (2009), pp. I4I-I58, pp. I5I-I52.

${ }_{27}$ Rodrigues, Elaine: «A imprensa pedagógica como fonte, tema e objeto para a história da educação», en Juvenal Costa, C.; Pereira Melo, J. J. y Hermenegildo Fabiano, L. (orgs.): Fontes e métodos em história da educação, Dourados, Universidade Federal de Grande Dourados, 20IO, pp. 3II-327, p. 312.

${ }_{28}$ Hernández Díaz, José María: «Prensa pedagógica y patrimonio histórico educativo en España. Conceptualización y géneros textuales», en Hernández Díaz, J. M. (ed.): Prensa pedagógica y patrimonio histórico educativo, Salamanca, Universidad de Salamanca, 2013, pp. 15-32, p. 28.

29 Checa Godoy, Antonio: Historia de la prensa pedagógica en España, Sevilla, Universidad de Sevilla, 2002. 
Hernanz. No es este el momento de hacer un análisis pormenorizado de la revista, pues ya hay algunos estudios recientes en los que se analiza como objeto de estudio y/o se utiliza como fuente de investigación ${ }^{30}$. Por ello, aportaremos, tan solo, algunos datos generales de la misma.

Escuelas de España está compuesta por un total de 44 ejemplares y aglutinó a un total de I64 personas. La obra se fundó en la provincia de Segovia, donde estuvo editándose hasta abril de 193I. En este momento, los maestros fundadores obtuvieron una plaza en el Patronato Escolar de Barcelona, por lo que la revista comenzó a editarse en la Ciudad Condal hasta enero de i932. Precisamente, este será al momento al que aludiremos en nuestro estudio. Sin embargo, la historia de la revista no termina aquí. Tras un periodo de inactividad, Escuelas de España reapareció e inauguró una segunda etapa en enero de 1934, esta vez desde Madrid, donde se estuvo publicando hasta julio de 1936, momento en el que se truncaron gran parte de las iniciativas de renovación pedagógica que habían aflorado durante el primer tercio del siglo en el país.

Entre las características principales de la revista destacamos tres: a) Su naturaleza aldeana, que se manifestó a través del respeto a la identidad rural y mediante las denuncias sobre el abandono que sufrían los docentes destinados en las escuelas rurales. b) Su carácter reformista, presente en las reivindicaciones de mejoras que conllevaban reformas que ellos mismos propusieron, comentaron, celebraron, cuestionaron y/o criticaron. Son foco de atención la reforma de la primera enseñanza, de las Escuelas Normales, de la inspección de primera enseñanza y del sistema de oposiciones. c) Su idiosincrasia innovadora y renovadora. La revista denota una probable influencia de la ILE. Asimismo, con una visión particular, caracterizada por el razonamiento y el análisis crítico, realista y superador, dedicaron profundas reflexiones a los nuevos métodos de enseñanza provenientes del extranjero. Los docentes valoraron esta nueva pedagogía, pero no pensaban que fuese la panacea que iba a resolver todos los problemas de la educación nacional;

30 Cachazo Vasallo, Alexia: «Escuelas de España. Barcelona (julio de I93I-enero de 1932)», en Hernández Díaz, J. M. (ed.): La prensa pedagógica de los profesores, Salamanca, Universidad de Salamanca, 20I8, pp. I4I-I5I; ORTIZ De SANTOS, Rosa: La renovación pedagógica desde la mirada de la revista Escuelas de España (1929-I93I) (Trabajo de Fin de Máster), Segovia, Universidad de Valladolid, 2017. Recuperado de https://cutt.ly/oaekfHQ; Ortiz De SAntos, Rosa: «La Universidad Popular Segoviana en la prensa pedagógica: Escuelas de España (1929-1936)», en Mora GARcía, J. L. y Sonlleva Velasco, M. (coords.): Educación, Cultura y Sociedad. Génesis y desarrollo de un proyecto reformista (colección La Universidad Popular Segoviana. Antecedentes, Historia y Protagonistas, tomo IV), Segovia, Real Academia de Historia y Arte de San Quirce, 2020, pp. 497- 519; OrTIZ De SANtos, Rosa, y Torrego Egido, Luis: «La prensa pedagógica en la reconstrucción del pasado educativo: el caso de la revista Escuelas de España (1929-1936)", Història da Educação, vól. 22, n. ${ }^{\circ} 56$ (2018), pp. 80-IO5. http://dx.doi.org/I0.1590/2236-3459/78399; Ortiz De Santos, Rosa y Torrego EGido, Luis: «Manuel Bartolomé Cossío en la revista pedagógica Escuelas de España (I929-1936)»,

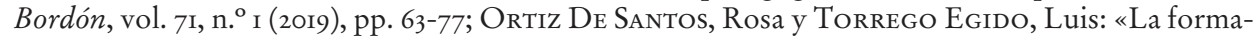
ción del profesorado en la revista pedagógica Escuelas de España (1929-1936)", en Libro en edición, Madrid, Dykinson, en prensa; Valdivieso Bermejo, Sara: Las Misiones Pedagógicas en Segovia: la visión de Escuelas de España (Trabajo de Fin de Grado), Segovia, Universidad de Valladolid, 2019. Recuperado de https://cutt.ly/chtHoya. 

ROSA ORTIZ DE SANTOS

reivindicaron la pedagogía española, defendiendo que esta habría de fortalecerse e incorporarse en el panorama pedagógico mundial.

El contenido de Escuelas de España es diverso; "aparecen contenidos de la pedagogía general, de organización y enseñanza de las diferentes disciplinas, de metodología educativa, así como biografías y problemas y reflexiones de la realidad educativa de la época» ${ }^{31}$. Uno de los temas que inquietó a nuestros redactores, por la actualidad que tenía, fueron los cuadernos de preparación de lecciones.

\subsection{Una preocupación de imperante actualidad: el cuaderno de preparación de lecciones}

En enero de 1932 , la revista pedagógica Escuelas de España publicó las respuestas de una sencilla encuesta que había realizado a un grupo de maestros de diferentes centros escolares de Barcelona y Zaragoza. En este momento, la revista se estaba publicando desde la capital catalana. Como ya avanzábamos en la presentación de la misma, los tres fundadores de la obra habían conseguido una plaza en el Patronato Escolar y, como ellos, la revista también tuvo que trasladarse a Barcelona. Desde allí, por tanto, se realizó la encuesta. Su principal motivación nos la explican los artífices de la misma:

Al poner en marcha los nuevos grupos escolares de Barcelona, se ha querido que los maestros lleven al día su cuaderno de preparación de lecciones. El asunto le consideramos tan importante y de tanta actualidad, por lo menos en Barcelona, que hemos querido saber la opinión de varios compañeros de esos grupos ${ }^{32}$.

Con ese propósito, formularon la siguiente pregunta: «¿Cuál es su opinión sobre el cuaderno de preparación de lecciones?». Utilizaron una única cuestión de carácter cualitativo, de respuesta libre y abierta. Obtuvieron la respuesta de 2I maestros (Cuadro I).

Cuadro i. Maestros encuestados

\begin{tabular}{|l|l|}
\hline Docentes de primera enseñanza & Centro educativo \\
\hline Dolores Soler Soler & \\
\cline { 1 - 1 } Antonio S. Muñoz & $\begin{array}{l}\text { Grupo Ramón Llull } \\
\text { Taimar }\end{array}$ \\
\cline { 1 - 1 } José María Tresánchez Camí & \\
\cline { 1 - 1 } José María Bonamusa Cascarra
\end{tabular}

31 Ortiz De Santos, Rosa y Torrego Egido, Luis: «La prensa pedagógica...», op. cit., p. ioo.

32 Escuelas DE EsPaÑA: «Una encuesta sobre el cuaderno de preparación de lecciones», Escuelas de España, primera época, n. ${ }^{\circ} \mathrm{I} 3$ (enero, I932), pp. 32-58, p. 32. 


\begin{tabular}{|l|l|}
\hline Ángel Ledesma Martín & $\begin{array}{l}\text { Grupo de Las Corts } \\
\text { (Barcelona) }\end{array}$ \\
\hline Mariano Rexach Fábrega & $\begin{array}{l}\text { Grupo Baixeras } \\
\text { (Barcelona) }\end{array}$ \\
\hline Ramón Casares Aisalá & \\
\hline Lorenzo Jou y Olió & \\
\cline { 1 - 1 } José Estruch Prim & \\
\hline Félix Martí Alpera & Grupo Pere Vila \\
\hline Miguel Espectante Calvo & (Barcelona) \\
\hline J. Millán & \\
\hline Aniceto Villar Villar & \\
\hline E. Ferrer y Gebelli & Grupo Escolar Valentín Zabala (Zaragoza) \\
\hline José María Andreu René
\end{tabular}

Fuente: Elaboración propia a partir de Escuelas de España (enero, 1932) ${ }^{33}$.

Entre los docentes encuestados localizamos importantes figuras de nuestra historia de la educación, como Félix Martí Alpera, partidario de la pedagogía activa, quien hizo una importante labor en la defensa de la escuela rural, impulsó las Sociedades Infantiles y fue pensionado por la Junta para la Ampliación de Estudios e Investigaciones Científicas (JAE). José María Pérez Civil fue un activo educador del magisterio aragonés. Lorenzo Jou y Olió, pensionado por la JAE, fue un miembro activo de la Federación Catalana de Maestros y el impulsor de «Les converses de Catalunya». José María Andreu René también recibiría una de las pensiones de la JAE para visitar instituciones educativas de Francia, Bélgica y Suiza. La valía de los demás profesionales queda retratada, además, por formar parte de los grupos escolares de Barcelona, cuyo enfoque pedagógico ha sido reconocido por la historiografía educativa.

Como veníamos explicando, Escuelas de España publicó las respuestas de los docentes, prometiendo a sus lectores que en el siguiente número realizarían un estudio donde se sintetizasen y comentasen las opiniones recibidas en su encuesta. Sin embargo, ese número no llegó a aparecer, pues la revista tuvo que suspender su publicación por razones económicas. No fue hasta enero de 1934 cuando resurgió desde Madrid, y dio inicio a su segunda etapa. El análisis de las respuestas de la encuesta nunca vio la luz. Desde esta pequeña aportación se pretende analizar los puntos de vista que los maestros encuestados por Escuelas

33 Ibidem. 
ROSA ORTIZ DE SANTOS

de España tenían acerca del cuaderno de preparación de lecciones, en un periodo de nuestra historia en el que estos, todavía, no eran obligatorios.

\section{Resultados de la encuesta: los cuadernos de preparación de lecciones}

Las opiniones sobre el cuaderno de preparación de lecciones fueron diversas. La mayoría de los encuestados coincidían en asociar aspectos tanto positivos como negativos a estos instrumentos, dependiendo de cómo se utilizasen, con qué finalidad y los principios pedagógicos sobre los que se sustentasen. Para facilitar el análisis y la síntesis de las respuestas, atendemos a aspectos clave y coincidentes en los discursos de los docentes.

\section{I. Un verdadero simbolo de la labor escolar}

Son pocos los docentes participantes en la encuesta que se posicionaron taxativamente a favor de los cuadernos de preparación de lecciones. Sin embargo, no faltaban opiniones en defensa de la eficacia y del valor que podían tener estos cuadernos para la mejora de la intervención educativa. Veamos un ejemplo en las palabras de Félix Martí Alpera: «Tan convencido estoy de la importancia del cuaderno de preparación de la labor escolar que le doy el valor de todo un símbolo» ${ }^{34}$. El maestro valoraba estos cuadernos, basándose en su experiencia, como una alternativa al libro de texto. Defendía que, a diferencia de este, el cuaderno del docente permitía adecuar los contenidos a las particularidades de los niños y de las niñas. Añadía que el cuaderno de preparación «es la ordenación de las ideas, de las cosas y de los trabajos. Es también la reflexión previa y la previsión que entrevé las peripecias, que se anticipa a las dificultades y que nos libra del azar sin renunciar a él» $»^{35}$.

No es el único que encontró beneficios en el diseño de estos instrumentos docentes. Federico Montserrat también pensaba que era un elemento imprescindible para la adecuación de los programas escolares a las capacidades de los discentes. José María Bonamusa, por otro lado, se expresaba en los siguientes términos: «Lo considero indispensable para todo Maestro que pretenda resultados positivos a la obra social que realiza [...] El cuaderno de preparación de lecciones refleja el alma y espíritu del Maestro» ${ }^{36}$. De igual manera, y según Antonio S. Muñoz, su importancia radicaba en que es un reflejo del trabajo realizado durante un curso. Con todo, no cabe duda de que su exigencia continuada requería «una atención y una perseverancia que ponen a prueba la buena voluntad del maestro» ${ }^{37}$, opinó Miguel Espectante.

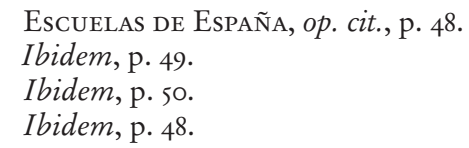




\section{2. "Ningún maestro, ni el más capacitado, está en condiciones de improvisar»}

La totalidad de los docentes que respondieron a la encuesta valoraban la preparación de las lecciones como una fase imprescindible para el buen funcionamiento de la labor escolar. «Una lección bien preparada tiene más probabilidades de fortuna que la espontánea» ${ }^{38}$, opinaba Lorenzo Jou y Olió. En este sentido, la gran mayoría reconocían que el cuaderno de preparación de lecciones podía ser un instrumento útil y eficaz que facilitaba la labor de planificación (Ramón Casares, Lorenzo Jou y Olió, Taimar, Francisco Manzanares, Antonio S. Muñoz, Miguel Espectante, José M. Andreu). Antonio S. Muñoz consideraba los cuadernos como una «garantía de seguridad para una lección provechosa» ${ }^{39}$. Por su parte, José M. Andreu explicaba que podía llegar a ser «muy útil para el maestro porque ha de servirle para concretar y enfocar la lección que va a dar y no se pierda en divagaciones y confusiones. Y muy eficaz para la enseñanza porque la simplifica, la metodiza y la hace más asequible para los alumnos» ${ }^{40}$.

Uno de los potenciales de los cuadernos más valorados por los participantes era que evitaban la improvisación como norma (Ramón Casares). El esfuerzo asociado a su desarrollo «está compensado [...] con creces, por la enorme ventaja que supone presentarse ante los alumnos sin tener que improvisar y con la seguridad de no vacilar en ningún instante» ${ }^{41}$, afirmaba Francisco Manzanares. José María Pérez Civil o José Estruch, quienes consideraban que la espontaneidad y la improvisación eran aspectos naturales, importantes y necesarios de la vida en el aula, opinaban, a su vez, que la labor de la escuela no podía ser puro azar.

\subsection{El cuaderno de preparación de lecciones desde una perspectiva práctica}

Muchas de las personas condicionaban su opinión sobre el cuaderno de preparación de lecciones en función de la manera en que este se concebía. Las características que deberían estar presentes en un cuaderno de preparación útil eran coincidentes en muchas de las respuestas. Los que se mostraban a favor citan estas características como necesarias y posibles de adecuar al cuaderno de preparación. Los que se posicionaban en contra citan las mismas características para criticar las carencias o peligros asociadas al instrumento docente. Taimar, por ejemplo y para empezar, pensaba que el cuaderno de preparación de lecciones era necesario si se planteaba con una finalidad práctica, con información precisa y presentada de forma esquemática.

Con esta idea coincidía José M. Andreu, quien defendía que «ese cuaderno de preparación no debe llevarse [...] con mucho detalle ni minuciosidad. Eso sería una ficción. Para mí, aquel cuaderno ha de ser un índice, un memento para el

${ }^{38} \quad$ Ibidem, p. 35 .

39 Ibidem, p. 46.

${ }^{40}$ Ibidem, p. 53.

${ }^{41}$ Ibidem, p. 45 . 

ROSA ORTIZ DE SANTOS

maestro en cada lección» ${ }^{42}$. José Estruch, en coherencia con sus compañeros, expresaba que «no hemos de pretender hacer [...] un tratado de didáctica y menos de literatura pedagógica» ${ }^{43}$. J. Millán también concebía el cuaderno de preparación como un instrumento de carácter práctico y, además, añadía que debía ser obra íntima del maestro; si se escapaba de esa intimidad, e incide en esto, sería un instrumento inútil y contraproducente.

Por otro lado, varios docentes, entre los que destaca José María Tresánchez, coincidían en que el cuaderno de preparación de lecciones, así como la propia planificación, esté o no desarrollada por escrito, para ser útil y práctica, debía ser abierta y flexible. Mariano Rexach añadía que «tomada de un modo rígido e invariable, sería ahora un estorbo atentatorio al máximo interés de la realidad presente» ${ }^{44}$.

Lorenzo Jou y Taimar se mostraban bastante escépticos con respecto a la aplicabilidad real de un cuaderno de preparación de lecciones. Creían que una lección podía estar bien trabajada sin la necesidad de prepararla por escrito, de forma ordenada y detallada. Asimismo, partiendo de que los docentes impartían numerosas lecciones por día, la elaboración de un cuaderno podría suponer dedicar más horas a preparar las sesiones por escrito que las que se empleaban en la propia práctica escolar. Sin embargo, sí valoraban la posibilidad de emplear otros instrumentos similares, con una naturaleza y mecánica diferentes. «Un libro de verdadera preparación de lecciones no es practicable. Lo que sí puede hacerse es llevar un libro de registro de las lecciones que se vayan dando durante el curso» ${ }^{45}$, opinaba Jou y Olió. Por su parte, Salvador Ruso defendía que este cuaderno debería denominarse «cuaderno de preparación del trabajo», dado que no atendía a una única disciplina, sino al ritmo y desarrollo de toda la labor educativa.

\subsection{La obligatoriedad de los cuadernos es una tortura para los maestros}

El cuaderno no debía ser obligatorio ni seguir normas preestablecidas. «En esta, como en otras muchas actividades, el maestro ha de gozar de libertad completa» ${ }^{46}$. Este era el pensamiento de una parte importante de los docentes encuestados. Taimar opinaba que «este cuaderno llevado sin exacción y sin pretensiones de realizar en él una obra acabada, puede rendir una gran utilidad, pero haciéndolo obligatorio es causa de tortura para el Maestro y de ningún valor para la enseñanza» ${ }^{47}$.

Mariano Rexach aconsejaba a los maestros y maestras «no caer en la estandarización de [s]u trabajo, que el Diario si no es flexible, dinámico, siempre nuevo, no

\footnotetext{
Ibidem, p. 53 .

Ibidem, p. 54 .

Ibidem, p. 56.

Ibidem, p. 36.

Ibidem, p. 38.

47 Ibidem, p. 39.
} 
cumple su misión ${ }^{48}$. Coincidía con él el maestro de Zaragoza José María Pérez Civil, quien tenía claro que «el cuaderno de preparación de lecciones debe tener por finalidad evitar en el hacer escolar el azar y la rutina. [...] Una orientación a seguir, pero dejando a salvo la libertad del maestro para desarrollar la lección según estime su sentido pedagógico» ${ }^{49}$.

Escribir los cuadernos bajo la presión de la obligatoriedad generaba lo que narra Aniceto Villar, y es que al final la mayoría de los cuadernos «parecen confeccionados a estilo burocrático, como cumpliendo una obligación formularia, a veces parecen ser un medio de salir del paso ${ }^{50}$. Desde esta perspectiva, y como explicaba Taimar, el cuaderno también perdía su sentido cuando se elaboraba «pensando en una posible inspección y crítica» ${ }^{51}$. En este caso, «el cuaderno representará una ficción, un engaño» ${ }^{52}$, y no reportará ningún beneficio a la escuela. Rexach también aludía a este problema, explicando que «si pensamos alguna vez en lo que dirán los demás de nuestro Diario de preparación, dejaremos de ser sinceros [...] a pretexto de una perfección engañosa ${ }^{53}$. En definitiva, y tomando las palabras de E. Ferrer y Gebelli, «el cuaderno de preparación de lecciones debe ser de uso exclusivo del maestro: en modo alguno ha de servir para deslumbrar a los profanos o a los pseudo-profesionales, ya que el tiempo de que dispone el maestro es tan limitado como precioso y no debe malgastarse en engañosos fuegos de artificio ${ }^{54}$.

\subsection{Lo esencial de la educación no puede reflejarse en ningún cuaderno}

Dolores Soler se manifestaba en contra de los cuadernos de preparación por una importante razón, que tenía clara, y es que la escuela y todo lo que esta conlleva no puede reflejarse de forma viva y natural en un cuaderno; «señalarle un cauce a la labor diaria de la Escuela, es quitarle esa ingenuidad y esa amplitud generosa que han de acompañar siempre la obra del niño y del Maestro» ${ }^{55}$, afirmaba. Desarrollar estos cuadernos, por tanto, significaría perturbar la obra educadora. Existía una parte de la labor escolar que no podía plasmarse en un cuaderno de preparación de lecciones, según Ramón Casares, «quizás lo más esencial, aquello que brota espontáneamente de la conversación del maestro con el alumno, necesariamente improvisación $»^{56}$.

\footnotetext{
Ibidem, p. 57 .

Ibidem, p. 46.

Ibidem, p. 40.

Ibidem, p. 37.

Ibidem, p. 38.

Ibidem, pp. 56-57.

Ibidem, p. 44.

Ibidem, p. 33.

Ibidem, p. 34 .
} 
Por otro lado, y en esta misma línea, Ángel Ledesma percibía que las exigencias del paso del tiempo obligaban al maestro a diseñar estos cuadernos, y no las necesidades naturales de la actividad escolar. Esto podría generar que la preparación de lecciones y, con ello, la propia práctica de aula, terminase convirtiéndose en una labor autómata, carente de sentido pedagógico. Y esto suponía una amenaza evidente para la buena educación. En coherencia con lo anterior, José María Pérez Civil, quien defendió que la educación no podía basarse únicamente en el azar, pensaba, a su vez, que

... la labor de la Escuela no puede ser mecánica. Toda labor mecánica es fría por carecer de alma que la vivifique. Un cuaderno de preparación de lecciones donde se contuvieran íntegras las lecciones con todo su desarrollo y toda la orientación a seguir, llevaría precipitadamente al maestro a la más extremada rutina, al más exagerado mecanismo ${ }^{57}$.

Mientras unos defendían la no mecanización de la labor docente, otros pensaban, por el contrario, que todo el proceso de preparación de lecciones debía estar perfectamente articulado. Es el caso de José M. Andreu, que declaraba: «Creo que el maestro debe llevar al día su cuaderno de preparación de lecciones ajustado al horario y al programa de su clase ${ }^{58}$. Esto conllevaba un riesgo que preocupaba a maestros como Ramón Casares o José Estruch: hacerse esclavo del cuaderno y perjudicar la espontaneidad. Estruch se manifestaba expresando que «tampoco queremos que el maestro se convierta en un esclavo [...]. Todos conocemos el valor de la enseñanza ocasional, y ésta no puede registrarla el 'Diario', porque no puede preverse» ${ }^{59}$.

\section{Conclusiones}

El cuaderno de preparación de lecciones es un claro precedente de las actuales unidades didácticas o programaciones escolares. Muchas de las reflexiones que hemos recuperado de la encuesta de Escuelas de España son, por tanto, de indudable actualidad. Quienes valoraban la conveniencia de desarrollar dichos cuadernos aludían a la peligrosidad y la imprudencia de basar toda la labor escolar en el azar y en la improvisación, y encontraban en los cuadernos de preparación un valioso auxiliar para la planificación de la labor escolar. Bien es cierto que la mayoría de los encuestados tenían una concepción similar de los cuadernos: los valoraban, desde una perspectiva práctica, como índice, y no como un desarrollo detallado de las sesiones. Al mismo tiempo, resaltaban su importancia por favorecer la adecuación de las sesiones a las peculiaridades de los escolares.

57 Ibidem, p. 45 .

$58 \quad$ Ibidem, p. 53.

59 Ibidem, p. 55 . 
Por otra parte, parece que existía consenso en que estos cuadernos deberían ser flexibles y abiertos. Para ello, resultaba indispensable que fuesen una obra personal e íntima del maestro, y que no estuviesen sometidos a normas externas estandarizadas. A esto, añadían su preocupación de que se convirtiese en una tarea obligatoria. Estos aspectos acabarían con el sentido pedagógico de los cuadernos. Asimismo, quienes se mostraron en contra de su aplicación real aludían a la imposibilidad de reflejar en un documento escrito aspectos del currículum oculto, quizás los más importantes de la acción educativa, así como el peligro de perjudicar la espontaneidad natural del aula, tan importante en los procesos de enseñanza-aprendizaje. ¿No son todas estas preocupaciones claramente aplicables a las programaciones docentes actuales?

Desde esta sencilla aportación nos hemos acercado a un instrumento importante de la cultura material escolar del primer tercio del siglo xx, como lo era el cuaderno de preparación de lecciones. Hemos percibido aspectos esenciales en su evolución a través del análisis de las opiniones que un nutrido grupo de maestros de escuela tenía sobre este recurso docente, cuando aún no eran de carácter obligatorio. El análisis en prensa de la época nos permite completar el vacío teórico que existe en la historiografía educativa acerca de los cuadernos de preparación. En el contexto que estudiamos, no podemos dejar de citar la pregunta que se hacían los fundadores de Escuelas de España en el ejemplar analizado: «¿Se llevará como cosa personal del maestro o como control para la fiscalización de su tarea? ${ }^{60}$. En aquel entonces, como hemos visto, tenía posibilidades para la mejora de la labor escolar para unos e importantes limitaciones para otros. No obstante, la intención inicial de su implementación tenía intereses que perseguían la mejora de la eficacia y de la calidad de la educación. Desconocían estos docentes que, años después, terminarían convirtiéndose en una imposición, en una obligación y en un instrumento de control, aspectos, todos ellos, limitadores de la libertad de los maestros y de las maestras.

Se abren nuevas incógnitas para el estudio de los cuadernos de preparación de lecciones, incluyendo el estudio de su evolución histórica, investigaciones histórico-educativas de los cuadernos de preparación de maestros y de maestras y su análisis comparativo con otros cuadernos de preparación de diferentes periodos históricos, o con respecto a las unidades didácticas y las programaciones docentes actuales. 